Mark McClure, Department of Mathematics, University of North Carolina at Asheville, Asheville, NC 28804, USA; e-mail: mcmcclur@bulldog.unca.edu

\title{
THE HAUSDORFF DIMENSION OF HILBERT'S COORDINATE FUNCTIONS
}

\begin{abstract}
We characterize the coordinate functions of Hilbert's space-filling curve using a directed-graph iterated function system and use this to analyze their fractal properties. In particular, we show that both coordinate functions have graphs of Hausdorff dimension $\frac{3}{2}$ and level sets of dimension $\frac{1}{2}$.
\end{abstract}

\section{Introduction}

Let $I=[0,1]$ denote the unit interval and let $I^{2}$ denote the unit square. Hilbert's space filling curve is a continuous, surjective function $h: I \rightarrow I^{2}$. The coordinate functions $x$ and $y$ are given by $h(t)=(x(t), y(t))$. An excellent general reference for $h$ is [7], where one may find arithmetical expressions for $x$ and $y$. More importantly, for this paper, are functional equations given in [7]. We use these to characterize the coordinate graphs using a directed-graph iterated functions system, henceforth referred to as a DiGraph IFS. This, in turn, allows us to show that the graphs of $x$ and $y$ have positive, finite $\frac{3}{2}$ dimensional Hausdorff measure. This is similar to the result in [5] that the coordinate functions of Peano's space filling curve are each self-affine, and also have positive, finite $\frac{3}{2}$-dimensional Hausdorff measure. Our techniques, however, more closely model those applied to Kiesswetter's curve in [1].

Let $X$ denote the graph of $x$ and let $Y$ denote the graph of $y$. We will see that $X$ may be decomposed into 4 parts: 2 affine images of itself and 2 affine images of $Y$. Similarly, $Y$ may be decomposed into 2 affine images of itself and 2 affine images of $X$. This is exactly the type of situation which may be described by a DiGraph IFS. Our treatment follows that of [6] and [2].

Key Words: space filling curves, Hausdorff dimension, self similarity

Mathematical Reviews subject classification: 26A27,28A30, 28A78

Received by the editors October 20, 1998 
A DiGraph IFS consists of a directed multi-graph, $G$, together with a function from $\mathbb{R}^{n}$ to $\mathbb{R}^{n}$ associated with each edge of $G$. We will assume that all of these functions are contractions, although this condition may be relaxed somewhat. The directed multi-graph, $G$, consists of a finite set, $V$, of vertices and a finite set, $E$, of directed edges. Given two vertices, $u$ and $v$, denote the set of all edges from $u$ to $v$ by $E_{u v}$. We denote the set of all paths of length $n$ with initial vertex $u$ by $E_{u}^{n}$. $G$ is called strongly connected if for every $u, v \in E$, there is a path from $u$ to $v$. Theorem 4.3.5 of [2] states that given any DiGraph IFS, there is a unique set of compact sets $K_{v}$, one for every $v \in V$, such that for every $u \in V$

$$
K_{u}=\bigcup_{v \in V, e \in E_{u v}} f_{e}\left(K_{v}\right)
$$

Such a set is called the invariant list of the DiGraph IFS. Note that if $e \in E_{u v}$, then $f_{e}$ maps $K_{v}$ into $K_{u}$. More generally, if $\alpha \in E_{u}^{n}$ has terminal vertex $v$, then we may form $f_{\alpha}: K_{v} \rightarrow K_{u}$ by composing the functions $f_{e}$ over $e \in \alpha$ taken in reverse order along the path $\alpha$. If $K$ is any compact set, then the sets $\bigcup_{\alpha \in E_{u}^{n}} f_{\alpha}(K)$ converge to $K_{u}$ in the Hausdorff metric as $n \rightarrow \infty$.

The sets $X$ and $Y$ will be characterized using affine functions. Define $A$ and $B$ to be the following matrices.

$$
A=\left(\begin{array}{cc}
\frac{1}{4} & 0 \\
0 & \frac{1}{2}
\end{array}\right) \quad B=\left(\begin{array}{cc}
\frac{1}{4} & 0 \\
0 & -\frac{1}{2}
\end{array}\right)
$$

Let $\vec{w} \in \mathbb{R}^{2}$ represent a column vector and define affine functions using matrix multiplication as follows.

$$
\begin{array}{ll}
a_{x x}(\vec{w})=A \vec{w}+\left(\begin{array}{c}
1 / 4 \\
0
\end{array}\right) & a_{y y}(\vec{w})=A \vec{w}+\left(\begin{array}{c}
1 / 4 \\
1 / 2
\end{array}\right) \\
b_{x x}(\vec{w})=A \vec{w}+\left(\begin{array}{c}
1 / 2 \\
1 / 2
\end{array}\right) & b_{y y}(\vec{w})=A \vec{w}+\left(\begin{array}{c}
1 / 2 \\
1 / 2
\end{array}\right) \\
c_{x y}(\vec{w})=A \vec{w} & c_{y x}(\vec{w})=A \vec{w} \\
d_{x y}(\vec{w})=B \vec{w}+\left(\begin{array}{c}
3 / 4 \\
1
\end{array}\right) & d_{y x}(\vec{w})=B \vec{w}+\left(\begin{array}{c}
3 / 4 \\
1 / 2
\end{array}\right)
\end{array}
$$

We may associate these functions to the edges in a DiGraph simply by labeling the edges. We may, also, label the vertices to indicate which one corresponds to $X$ and which one corresponds to $Y$. The labeled DiGraph for $X$ and $Y$ is shown in figure 1.

Lemma 1.1. $X$ and $Y$ form the invariant list of the DiGraph IFS shown in figure 1. 


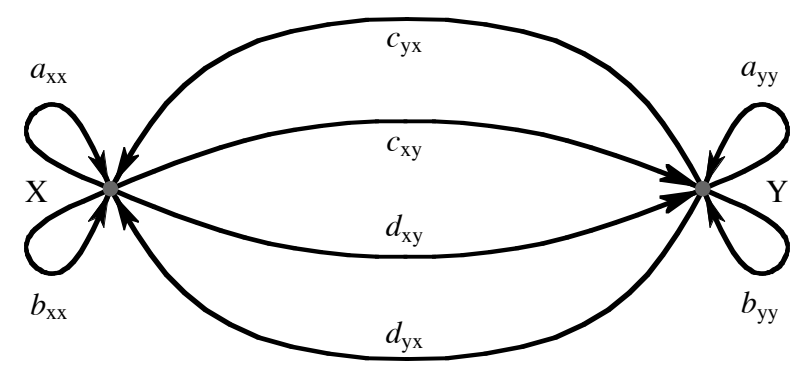

Figure 1: The DiGraph for $X$ and $Y$

Proof. This follows immediately from the fact that $x$ and $y$ satisfy the following list of functional equations ([7], page 30, ex. 13). We have labeled the functional equations to clarify the correspondence.

$$
\begin{array}{ll}
a_{x x}: x((1+t) / 4)=x(t) / 2 & a_{y y}: y((1+t) / 4)=1 / 2+y(t) / 2 \\
b_{x x}: x((2+t) / 4)=1 / 2+x(t) / 2 & b_{y y}: y((2+t) / 4)=1 / 2+y(t) / 2 \\
c_{x y}: x(t / 4)=y(t) / 2 & c_{y x}: y(t / 4)=x(t) / 2 \\
d_{x y}: x((3+t) / 4)=1-y(t) / 2 & d_{y x}: y((3+t) / 4)=1 / 2-x(t) / 2
\end{array}
$$

\section{Hausdorff Measure and Dimension}

In this section, we recall the definitions of Hausdorff measure and dimension, show that $X$ and $Y$ have dimension $\leq 3 / 2$, and state some useful lemmas. Our notation has been influenced by [2] and [4], where one may find proofs of the basic facts.

Let $s \geq 0$. We will define the $s$-dimensional Hausdorff measure, $\mathcal{H}^{s}$, on Euclidean space, $\mathbb{R}^{n}$. Let $F \subset \mathbb{R}^{n}$. The diameter of $F$ will be denoted by $\operatorname{diam}(F)$. Let $\varepsilon>0$. An $\varepsilon$-cover, $\mathcal{C}$, of $F$ is a countable collection of sets such that $F \subset \cup_{U \in \mathcal{C}} U$ and $\operatorname{diam}(U) \leq \varepsilon$ for every $U \in \mathcal{C}$. Now define

$$
\mathcal{H}_{\varepsilon}^{s}(F)=\inf \left\{\sum_{U \in \mathcal{C}} \operatorname{diam}(U)^{s}: \mathcal{C} \text { is an } \varepsilon \text {-cover of } F\right\}
$$

and

$$
\mathcal{H}^{s}(F)=\lim _{\varepsilon \rightarrow 0^{+}} \mathcal{H}_{\varepsilon}^{s}(F) .
$$

Note that this limit is well defined since $\mathcal{H}_{\varepsilon}^{s}(F)$ increases as $\varepsilon$ decreases. It may be shown that $\mathcal{H}^{s}$ is a Borel outer measure on $\mathbb{R}^{n}$. We denote its restriction to 
the $\mathcal{H}^{s}$-measurable sets by $\mathcal{H}^{s}$, also, and call this the $s$-dimensional Hausdorff measure.

The Hausdorff dimension of $F$, $\operatorname{dim}(F)$, is defined by

$$
\operatorname{dim}(F)=\inf \left\{s \geq 0: \mathcal{H}^{s}(F)=0\right\} .
$$

If $F$ is an infinite set, this is equivalent to

$$
\operatorname{dim}(F)=\sup \left\{s \geq 0: \mathcal{H}^{s}(F)=\infty\right\} .
$$

We will show that $0<\mathcal{H}^{3 / 2}(X)<\infty$, so that $\operatorname{dim}(X)=3 / 2$, and similarly for $Y$. We prove the upper bound here and the lower bound in the next section.

Theorem 2.1. $\mathcal{H}^{3 / 2}(X) \leq 2^{3 / 4}$, so $\operatorname{dim}(X) \leq 3 / 2$. A similar statement holds for $Y$.

Proof. We write the proof for $X$. The proof for $Y$ is identical. Note that each of the affine functions in the DiGraph IFS defining $X$ and $Y$ maps $I^{2}$ into $I^{2}$. Thus $X_{n}=\bigcup_{\alpha \in E_{X}^{n}} f_{\alpha}\left(I^{2}\right)$ forms a nested sequence of sets containing the invariant set $X$. Furthermore, each set $f_{\alpha}\left(I^{2}\right)$ is a rectangle with width $4^{-n}$ and height $2^{-n}$, due to the affine nature of the functions. There are $4^{n}$ of these sets since there are $4^{n}$ paths of length $n$ leaving any vertex in the DiGraph.

Now, each of the rectangles $f_{\alpha}\left(I^{2}\right)$ may be decomposed into $2^{n}$ squares of side length $4^{-n}$. Thus, we may cover $X$ by $2^{n} 4^{n}$ squares of side length $4^{-n}$. Therefore,

$$
\mathcal{H}_{\sqrt{2} 4^{-n}}^{3 / 2}(X) \leq 2^{n} 4^{n}\left(\sqrt{2} 4^{-n}\right)^{3 / 2}=2^{3 / 4}
$$

and $\mathcal{H}^{3 / 2}(X) \leq 2^{3 / 4}$, as $n$ is arbitrary.

Lower bounds for Hausdorff measure are, typically, more difficult. Our strategy will be to show that $\mathcal{H}^{1 / 2}\left(x^{-1}(z)\right)>0$ for all $z \in[0,1]$. The lower bound for $X$ will then follow from a result of Besicovitch. We will obtain the lower bound for level sets by using following measure comparison lemma ([4], page 55).

Lemma 2.1. Let $\mu$ be a Borel measure on the Borel set $F$ and suppose that for some $s>0$, there are numbers $c, \delta>0$ such that $\mu(U) \leq c \operatorname{diam}(U)^{s}$ for all open sets $U$ with $\operatorname{diam}(U) \leq \delta$. Then, $\mathcal{H}^{s}(F) \geq \mu(F) / c$.

We will, also, need the following scaling property of Hausdorff measure ([4], page 27). 
Lemma 2.2. If $F \subset \mathbb{R}^{n}, \lambda>0$, and $\lambda F=\{\lambda x: x \in F\}$, then $\mathcal{H}^{s}(\lambda F)=$ $\lambda^{s} \mathcal{H}^{s}(F)$.

Finally, we will need Mauldin and Williams' computation of the Hausdorff measure of DiGraph self-similar sets. We associate a similarity dimension with any DiGraph IFS with similarities as follows. Suppose that for each $e \in E$, $f_{e}$ is a similarity with ratio $r_{e}$. Construct a matrix $M(s)$ whose rows and columns are indexed by the vertex set $V$. The element in row $u$ and column $v$ is $\sum_{e \in E_{u v}} r_{e}^{s}$. The similarity dimension of the DiGraph IFS is the unique value of $s$ such that $M(s)$ has spectral radius 1. This coincides with the Hausdorff dimension of the corresponding DiGraph self-similar sets, provided an open set condition is satisfied. The open set condition states that there should be open sets $U_{v}$, one for each $v \in V$, so that $U_{u} \supset \bigcup_{e \in E_{u v}} f_{e}\left(U_{v}\right)$ with this union disjoint. The following is the main result of [6]. See, also, [2] theorem 6.4.8.

Lemma 2.3. Let $\left\{K_{v}\right\}_{v \in V}$ be the invariant list of a self-similar DiGraph IFS arising from a strongly connected directed multi-graph and with similarity dimension s. Then $\mathcal{H}^{s}\left(K_{v}\right)<\infty$ for all $v \in V$. If, in addition, the open set condition is satisfied, then $\mathcal{H}^{s}\left(K_{v}\right)>0$ for all $v \in V$.

Note that self-similarity is the special case of DiGraph self-similarity where the DiGraph has one vertex. In this case, lemma 2.3 reduces to the standard formula for similarity dimension.

\section{The Structure of Level Sets}

We now turn our attention to the structure of level sets. We first consider the sets $x^{-1}(0), y^{-1}(0), x^{-1}(1)$, and, $y^{-1}(1)$ and show they all have Hausdorff dimension 1/2. Then, we will use the DiGraph structure of $X$ and $Y$ to extend these results to other sets.

Consider the functions $a_{x x}, c_{x y}, c_{y x}$, and $d_{y x}$. These are the four affine transformations from figure 1 which leave the $x$-axis invariant. They are all similarities of ratio $\frac{1}{4}$ when restricted to $\mathbb{R}$. Thus, the sets $x^{-1}(0)$ and $y^{-1}(0)$ form the invariant list of the corresponding self-similar DiGraph IFS. The open set condition is satisfied using the open unit interval. Lemma 2.3 shows that

$$
0<\mathcal{H}^{1 / 2}\left(x^{-1}(0)\right)<\infty \quad \text { and } \quad 0<\mathcal{H}^{1 / 2}\left(y^{-1}(0)\right)<\infty .
$$

In fact, note that $\mathcal{H}^{1 / 2}\left(x^{-1}(0)\right) \leq 1$ since $x^{-1}(0)$ may be covered by $2^{n}$ intervals of length $4^{-n}$ for any $n$. A similar statement holds for $y$. 
We need to highlight a certain regularity in these sets in order to extend results to other sets.

Lemma 3.1. $\mathcal{H}^{1 / 2}\left(x^{-1}(0) \cap U\right) \leq 4 \operatorname{diam}(U)^{1 / 2}$ for all Borel sets $U \subset I$. A similar statement holds for $y$.

Proof. First, consider the case where $U$ is a closed interval of the form

$$
\left[i / 4^{n},(i+1) / 4^{n}\right] \text { where } i, n \in \mathbb{N} \text { and } 0 \leq i<4^{n} .
$$

Then, either $\mathcal{H}^{1 / 2}\left(x^{-1}(0) \cap U\right)=0$ or $x^{-1}(0) \cap U$ is a set similar to $x^{-1}(0)$ or $y^{-1}(0)$ scaled by a factor $4^{-n}$. In either case,

$$
\mathcal{H}^{1 / 2}\left(x^{-1}(0) \cap U\right) \leq\left(4^{-n}\right)^{1 / 2} \mathcal{H}^{1 / 2}\left(x^{-1}(0)\right)=\operatorname{diam}(U)^{1 / 2} .
$$

Now, suppose that $U$ satisfies $4^{-(n+1)} \leq \operatorname{diam}(U)<4^{-n}$. Then, $U$ may be covered by at most 2 intervals of the form $\left[i / 4^{n},(i+1) / 4^{n}\right]$. Thus,

$$
\mathcal{H}^{1 / 2}\left(x^{-1}(0) \cap U\right) \leq 2\left(4^{-n}\right)^{1 / 2}=4\left(4^{-(n+1)}\right)^{1 / 2} \leq 4 \operatorname{diam}(U)^{1 / 2} .
$$

Next, consider the sets $x^{-1}(1)$ and $y^{-1}(1)$. We see that $x^{-1}(1)$ is isometric to $x^{-1}(0)$ by using another of Sagan's functional equations: $x(t)+x(1-t)=1$ ([7], ex. 13, page 30). In particular, if $t$ satisfies $x(t)=0$, then $1-t$ satisfies $x(1-t)=1$. Therefore, $0<\mathcal{H}^{1 / 2}\left(x^{-1}(1)\right) \leq 1$ and $x^{-1}(1)$ satisfies the conclusions of lemma 3.1.

Finally, $y^{-1}(1)$ is a self-similar set for the similarities $a_{y y}$ and $b_{y y}$ restricted to the horizontal line $y=1$. Again, $0<\mathcal{H}^{1 / 2}\left(y^{-1}(1)\right) \leq 1$ and $y^{-1}(1)$ satisfies the conclusions of lemma 3.1.

We now consider the extension to other level sets.

Lemma 3.2. Suppose that $n \in \mathbb{N}$ and that $j$ is an odd integer satisfying $1 \leq j<2^{n}$. Then, $x^{-1}\left(j / 2^{n}\right)$ and $y^{-1}\left(j / 2^{n}\right)$ both consist of $2^{n+1}$ sets which are similar to one of the basic sets $x^{-1}(0), y^{-1}(0), x^{-1}(1)$, or, $y^{-1}(1)$, scaled by a factor $4^{-n}$.

Proof. First, note that the result is true for $n=1$, as $x^{-1}(1 / 2)$ consists of a copy of $y^{-1}(1)$ over $[0,1 / 4]$, a copy of $x^{-1}(1)$ over [1/4,1/2], a copy of $x^{-1}(0)$ over $[1 / 2,3 / 4]$, and a copy of $y^{-1}(1)$ over $[3 / 4,1]$. This may be seen from the action of the DiGraph IFS. Similarly, $y^{-1}(1 / 2)$ consists of a copy of $x^{-1}(1), 2$ copies of $y^{-1}(0)$, and a copy of $x^{-1}(0)$.

Proceeding by induction, suppose the result is true for $n \in \mathbb{N}$. Let $j$ be an odd integer satisfying $1 \leq j<2^{n+1}$. 
Case 1: $j<2^{n}$. Then, $x^{-1}\left(j / 2^{n+1}\right)$ consists of a copy of $y^{-1}\left(j / 2^{n}\right)$ over $[0,1 / 4]$ and a copy of $x^{-1}\left(j / 2^{n}\right)$ over [1/4,1/2], each scaled by a factor $1 / 4$.

Case 2: $j>2^{n}$. Then, $x^{-1}\left(j / 2^{n+1}\right)$ consists of a copy of $x^{-1}\left(1-j / 2^{n}\right)$ over $[1 / 2,3 / 4]$ and a copy of $y^{-1}\left(j / 2^{n}\right)$ over $[3 / 4,1]$, each scaled by a factor $1 / 4$.

In both cases, the induction hypotheses shows that we have a total of $2^{n+2}$ copies of the basic sets scaled by a factor $4^{-(n+1)}$. A similar argument applies to $y$.

Now, let

$$
m=\min \left\{\mathcal{H}^{1 / 2}\left(x^{-1}(0)\right), \mathcal{H}^{1 / 2}\left(y^{-1}(0)\right), \mathcal{H}^{1 / 2}\left(x^{-1}(1)\right), \mathcal{H}^{1 / 2}\left(y^{-1}(1)\right)\right\} .
$$

Corollary 3.1. If $z$ is a dyadic rational and $U$ is a Borel set, then

$$
m \leq \mathcal{H}^{1 / 2}\left(x^{-1}(z)\right) \leq 2
$$

and

$$
\mathcal{H}^{1 / 2}\left(x^{-1}(z) \cap U\right) \leq 4 \operatorname{diam}(U)^{1 / 2} .
$$

Proof. This follows immediately from the scaling lemma 2.2 and lemma 3.2.

The fact that $\mathcal{H}^{1 / 2}\left(x^{-1}(z)\right)>0$ for all $z \in I$ now follows from the following lemma, which generalizes a technique applied to Kiesswetter's curve by Edgar.

Lemma 3.3. Let $s>0$ and let $f$ be a continuous, real valued function defined on some closed interval J. Suppose there are numbers $a$ and $b$ such that

$$
0<a \leq \mathcal{H}^{s}\left(f^{-1}(z)\right) \leq b
$$

for all $z$ in some dense subset $D \subset$ range $(f)$. Suppose further that there is a $c>0$ such that for all $z \in D$ and for all open sets $U$ we have

$$
\mathcal{H}^{s}\left(f^{-1}(z) \cap U\right) \leq c \operatorname{diam}(U)^{s} .
$$

Then, $\mathcal{H}^{s}\left(f^{-1}(z)\right) \geq a / c$ for all $z \in \operatorname{range}(f)$.

Proof. Fix $z \in$ range $(f)$ and choose a sequence $\left(z_{n}\right)$ from $D$ such that $z_{n} \neq z$ for any $n$, and $z_{n} \rightarrow z$ as $n \rightarrow \infty$. For each $n \in \mathbb{N}$, define the Borel measure $\mu_{n}$ on $J$ to be $\mu_{n}=\left.\mathcal{H}^{s}\right|_{f^{-1}\left(z_{n}\right)}$, the restriction of $\mathcal{H}^{s}$ to $f^{-1}\left(z_{n}\right)$. Since $a \leq \mu_{n}(J) \leq b$ for every $n$, this sequence has some weak-* cluster point, say $\mu$, satisfying $a \leq \mu(J) \leq b$.

We claim that $\mu$ is supported on $f^{-1}(z)$. Suppose $U$ is an open set containing $f^{-1}(z)$. Then there is an open set $V$ such that $f^{-1}(z) \subset V \subset \bar{V} \subset U$. By the continuity of $f$, we have $f^{-1}\left(z_{n}\right) \subset V$ for large enough $n$. Thus,

$$
\mu(J \backslash U) \leq \mu(J \backslash \bar{V}) \leq \liminf _{n \rightarrow \infty} \mu_{n}(J \backslash \bar{V})=0
$$


and $\mu\left(J \backslash f^{-1}(z)\right)=0$. Furthermore, if $U \subset J$ is any open set, then

$$
\mu(U) \leq \liminf _{n \rightarrow \infty} \mathcal{H}^{s}\left(f^{-1}\left(z_{n}\right) \cap U\right) \leq c \operatorname{diam}(U)^{s} .
$$

Thus, $\mathcal{H}^{s}\left(f^{-1}(z)\right) \geq a / c$ by lemma 2.1 .

Corollary 3.2. $\mathcal{H}^{1 / 2}\left(x^{-1}(z)\right) \geq m / 4>0$ and $\mathcal{H}^{1 / 2}\left(y^{-1}(z)\right) \geq m / 4>0$ for all $z \in I$.

Proof. Simply combine corollary 3.1 and lemma 3.3.

The following lemma is Theorem 5.8 of [3], but is originally due to Besicovitch. We will need it to transfer results to $X$ and $Y$. If $F \subset \mathbb{R}^{2}$, where $\mathbb{R}^{2}$ is the $x z$ plane, then $F_{z}=\{x \in \mathbb{R}:(x, z) \in F\}$ represents a level set.

Lemma 3.4. Let $F$ be a subset of the $x z$ plane and let $A$ be any subset of the $z$-axis. Suppose that if $z \in A$, then $\mathcal{H}^{t}\left(F_{z}\right)>c$, for some constant $c$. Then

$$
\mathcal{H}^{s+t}(F) \geq b c \mathcal{H}^{s}(A),
$$

where $b$ depends only on $s$ and $t$.

Corollary 3.3. $\mathcal{H}^{3 / 2}(X)>0$ and $\mathcal{H}^{3 / 2}(Y)>0$.

Proof. This follows immediately from corollary 3.2 and lemma 3.4 by taking $A$ to be $[0,1], t=1 / 2$, and $s=1$.

\section{Comments}

We have proved that $0<\mathcal{H}^{3 / 2}(X)<\infty$ and similarly for $Y$. More that this, we have obtained the stronger fact that $0<\mathcal{H}^{1 / 2}\left(x^{-1}(z)\right)$ for all $z \in[0,1]$. Not only does this imply that $0<\mathcal{H}^{3 / 2}(X)$, but the reverse implication is not true in general. Indeed, all of the vertical cross-sections of $X$ are singletons and, therefore, zero dimensional.

The fact that $\mathcal{H}^{3 / 2}(X)<\infty$, implies $\mathcal{H}^{1 / 2}\left(x^{-1}(z)\right)<\infty$ for almost all $z \in[0,1]$. This statement is easily improved. Consider the rectangular covers of $X$ used in the proof of theorem 2.1. One may prove by induction that any horizontal line intersects at most $2 \cdot 2^{n}$ of the rectangles of width $4^{-n}$. Thus, for any $z \in[0,1]$,

$$
\mathcal{H}_{4^{-n}}^{1 / 2}\left(x^{-1}(z)\right) \leq 2 \cdot 2^{n}\left(4^{-n}\right)^{1 / 2}=2
$$

and $\mathcal{H}^{1 / 2}\left(x^{-1}(z)\right) \leq 2$ since $n$ is arbitrary.

\section{Acknowledgments}

The referees and editor provided many useful comments to improve this paper. 


\section{References}

[1] G. A. Edgar, "Kiesswetter's fractal has Hausdorff dimension 3/2." The Real Analysis Exchange 14 (1988-89), 215-223.

[2] G. A. Edgar, Measure, Topology, and Fractal Geometry. Springer-Verlag, New York, 1990.

[3] K. J. Falconer, The Geometry of Fractal Sets. Cambridge University Press, Cambridge, England, 1985

[4] K. J. Falconer, Fractal Geometry: Mathematical Foundations and Applications. John Wiley and Sons, West Sussex, England, 1990.

[5] Norio Kono, "On self-affine functions." Japan J. Appl. Math. 3 (1986), 259-269.

[6] R. D. Mauldin and S. C. Williams, "Hausdorff dimension in graph directed constructions." Trans. Amer. Math. Soc. 309 (1988) 811-829.

[7] Hans Sagan, Space-Filling Curves. Springer-Verlag, New York, 1994. 